\author{
laralice Medeiros de Souza ${ }^{1}$ \\ Milton Neto da Silva ${ }^{1}$ \\ Elza Scott Figueira ${ }^{1}$ \\ Maria de Lourdes Leal ${ }^{1}$ \\ Ellen Jessouroun ${ }^{1}$ \\ Shirley de Mello Pereira \\ Abrantes $^{2}$ \\ Ivna Alana Freitas \\ Brasileiro da Silveira ${ }^{1}$ \\ ${ }^{1}$ Laboratório de Tecnologia \\ Bacteriana, Bio-Manguinhos, \\ Fundação Oswaldo Cruz, Rio de \\ Janeiro, Brazil \\ ${ }^{2}$ Laboratório de Alimentos e \\ Contaminantes, Departamento \\ de Química, Instituto Nacional \\ de Controle de Qualidade em \\ Saúde, Fundação Oswaldo Cruz, \\ Rio de Janeiro, Brazil
}

Received July 1, 2013

Revised August 5, 2013

Accepted August 5, 2013
Research Article

\section{Single validation of CE method for determining free polysaccharide content in a Brazilian meningococcal $\mathrm{C}$ conjugate vaccine}

\begin{abstract}
Neisseria meningitidis group $\mathrm{C}$ is an encapsulated bacterium that causes several diseases and is associated with high mortality rates, thereby constituting a serious public health problem. Bio-Manguinhos/Fiocruz is developing a conjugate vaccine by covalent attachment of capsular polysaccharide to hydrazide-activated tetanus toxoid through reductive amination. It is necessary to quantify free components as a quality control process to prevent exacerbated adverse reactions and/or attenuation of vaccine immunogenicity. Thus, this study aimed to develop and validate a quality control method appropriate for the separation and quantification of free polysaccharide present in this conjugate $N$. meningitidis group $\mathrm{C}$ vaccine using CE. CZE was used to remove unbound polysaccharide, and the electrophoretic conditions were varied to optimize resolution. We were able to develop and validate the proposed method, which was linear and showed a matrix effect. Repeatability and partial reproducibility of the method were also evaluated. The robustness results showed that control of temperature is required for reliable results. The validated method will be used to evaluate the conjugate batches submitted for Phase III clinical studies and for routine quality control of the conjugate vaccine.
\end{abstract}

\section{Keywords:}

Capillary electrophoresis / Meningococcal vaccine / Polysaccharide content / Single validation

DOI 10.1002/elps.201300298

\section{Introduction}

Neisseria meningitidis is a Gram-negative diplococcus that colonizes the pharynx and upper respiratory tract. Thirteen groups have been identified based on unique capsular polysaccharide (PS) antigen compositions. Six of these (A, B, C, W-135, X, and Y) are responsible for almost all human diseases. The majority of meningococcal disease in Europe and the Americas are associated with groups B and C, whereas groups $\mathrm{A}$ and $\mathrm{C}$ are prevalent in Asia and Africa.

In Brazil, meningococcal disease is endemic, with sporadic cases occurring throughout the year. Groups B and $\mathrm{C}$ predominate; however, group $\mathrm{C}$ has been associated

Correspondence: laralice Medeiros de Souza, Laboratório de Tecnologia Bacteriana, Bio-Manguinhos, Fundação Oswaldo Cruz, Av. Brasil 4365, Manguinhos, Pavilhão Rockfeller $3^{\circ}$ Andar. Rio de Janeiro, CEP: 21040-900, Brazil

E-mail: iaralice.medeiros@bio.fiocruz.br

Fax: $+21-2260-4727$

Abbreviations: CPA, corrected peak area; $\mathbf{N a O H}$, sodium hydroxide; $\mathbf{P S}$, polysaccharide; $\mathbf{S}_{\mathbf{i}}$, intermediary standard deviation; TBNa, sodium tetraborate buffer; TT, tetanus toxoid; WHO, World Health Organization with outbreaks in different regions of the country. BioManguinhos/Fiocruz has recently produced a PS vaccine against groups A, C, and W-135 in collaboration with the Finlay Institute of Cuba. However, PS vaccines are known to be less immunogenic because they do not elicit a $\mathrm{T}$ celldependent immune response and are ineffective in protecting children less than two years of age. Therefore, BioManguinhos, the unit within the Oswaldo Cruz Foundation involved in the production of immunobiologicals, has been developing a conjugate vaccine against group $\mathrm{C}$ meningococcus using tetanus toxoid (TT) as the carrier protein for several years [1]. The World Health Organization (WHO) recommends maximum limits for the free components, protein and PS, remaining after conjugation [2]. The free oxidized PS yield determines the process efficiency, but the WHO guidelines do not specify the maximum concentration of this component in meningococcal $\mathrm{C}$ conjugate vaccine.

Several methods have been described for the quantification of the PS content in the meningococcal conjugate vaccine [3-5]. Most of these use detergents such as CTAB and DOC for precipitating PS and quantify PS using colorimetric and chromatographic methods [3,4]. Other techniques employ the reaction of the antibody against TT to remove free PS [5]. The majority of these physicochemical methods are difficult to validate because they require some extraction and 
sample preparation steps before analysis. CE is a widely used technique and has been useful for separating and identifying many compounds with a high resolution. In the field of conjugate vaccine, this technique has been used for the quality control of meningococcal and pneumococcal vaccines to determine the free protein content [6]. PS from different meningococcal groups has also been separated and quantified using this technique [7]. CZE and MEKC are the major techniques that have been used for studying vaccines.

The aim of the present study was to determine the free oxidized PS content present in the Brazilian meningococcal conjugate vaccine after separation and quantification using CE. The method developed is simple to perform and does not require sample pretreatment. Further, an intralaboratory validation was conducted according to the guidelines of the Agência Nacional de Vigilância Sanitária (ANVISA, National Sanitary Surveillance Agency) and Instituto Nacional de Metrologia, Qualidade e Tecnologia (INMETRO, National Institute of Metrology, Quality, and Technology).

\section{Materials and methods}

\subsection{Samples}

\subsubsection{Oxidized PS}

Native PS was produced from the vaccine strain 2135 of $N$. meningitidis group $\mathrm{C}$ and was purified and analyzed according to the procedures described for meningococcal vaccine production $[3,8]$. Oxidized PS was produced by reacting native PS with $23.4 \mathrm{mM}$ sodium periodate at $4^{\circ} \mathrm{C}$ as described previously [1]. Tangential flow ultrafiltration was used in the purification process (Centrasette, Pall BioPharmaceuticals) to remove unreacted reagents [9]. For CE, oxidized PS was diluted in water to $1 \mathrm{mg} / \mathrm{mL}$. To determine the working concentration, PS samples were analyzed using a spectrophotometric method [10].

\subsubsection{Conjugate bulk}

The conjugate bulks were prepared by forming covalent bind between the aldehyde groups of oxidized PS and the hydrazide groups generated in the protein (TT, produced by Butantan Institute, São Paulo, Brazil). The reaction was performed as described by Silveira et al. [1] using sodium cyanoborohydride as a reducing agent. Upon completion of the reaction, all bulks were diluted with $1 \mathrm{mM}$ phosphate buffer, pH 7.0. Tangential flow ultrafiltration was used in the purification process (Centrasette, Pall BioPharmaceuticals) to remove unreacted reagents, by-products, and unconjugated oxidized PS [9]. For CE, the concentration of conjugate bulk prepared by Bio-Manguinhos/Fiocruz must be in the range of approximately $1-3 \mathrm{mg} / \mathrm{mL}$ protein and $1-2 \mathrm{mg} / \mathrm{mL}$ PS. The PS content was determined using a spectrophotometric method [10].

\subsection{Instrumentation}

Free oxidized PS was purified and quantified using a CE system (HP 3D CE), with a photodiode array detector equipped with forced air temperature control, platinum electrodes, and ChemStation Software G1601A for instrument control and data analysis. A fused silica capillary coated with polyimide, $112.5 \mathrm{~cm}$ in length, effective size of $104 \mathrm{~cm}$, and $50 \mu \mathrm{m}$ in inner diameter (Agilent Technologies, USA) was used. CZE was chosen for PS separation based on the molecular characteristics, size, and charge [11,12]. A wavelength of $200 \mathrm{~nm}$ was used for these experiments.

\subsection{Reagents and experimental conditions}

Separation buffer was freshly prepared on the day of use by mixing $50 \mathrm{mM}$ of sodium tetraborate (TBNa) with Milli-Q purified water. The $\mathrm{pH}$ was adjusted to 10 with $1 \mathrm{M}$ sodium hydroxide $(\mathrm{NaOH})$. The capillary was rinsed with $1 \mathrm{M} \mathrm{NaOH}$, Milli-Q purified water, and separation buffer for 10, 10, and $30 \mathrm{~min}$, respectively. The capillary was rinsed prior to each separation with $0.1 \mathrm{M} \mathrm{NaOH}$ and water for $3 \mathrm{~min}$ each and with separation buffer for $5 \mathrm{~min}$.

\subsection{Method development}

The initial separation of conjugate bulk using CE incorporated some of the conditions described by Lamb et al. [7] as follows: $20 \mathrm{mM}$ TBNa buffer, $\mathrm{pH} 9.3,72 \mathrm{~cm}$ fused silica capillary. However, several studies were required for optimizing parameters, such as capillary length, buffer concentration, $\mathrm{pH}$, temperature, voltage, and current, until the final conditions were established (data not shown) as follows: $50 \mathrm{mM}$ TBNa buffer, $\mathrm{pH} 10,30 \mathrm{kV}, 40^{\circ} \mathrm{C}$, and $104 \mathrm{~cm}$ effective capillary length.

\subsection{Selectivity}

The free oxidized PS peak was identified by fortifying the conjugate with oxidized PS and comparing the migration times of the peak observed in the sample with that of oxidized PS alone. In addition, the migration times of other components, conjugate, and protein that could interfere were evaluated.

\subsection{Intralaboratory validation}

The method was validated according to the parameters recommended by Brazilian regulatory agencies $[13,14]$.

\subsubsection{Linearity}

Concentrations of oxidized PS used to establish the calibration line were determined according to those recommended by WHO for the Haemophilus influenzae type b conjugate 
vaccine. This vaccine was the first for which the limit of free sugar (20\%) was described [15]. From this limit, a calibration line was developed using seven concentrations of PS varying in uniform steps from 5 to $35 \%$ with range of $5 \%$. The central point concentration of the curve was equivalent to the maximum expected concentration of free sugar in conjugate vaccines. Solutions were prepared from two stock solutions of oxidized PS and diluted in Milli-Q water to determine if the concentration line was linear using the corrected peak area (CPA).

\subsubsection{Matrix effect evaluation}

To evaluate if the matrix interferes with the quantification of PS, the calibration line was generated using the matrix (the conjugate bulk sample) as the solvent instead of buffer. Therefore, we added the same amounts of oxidized PS tested for linearity to the conjugate sample and determined the variation in homogeneity for the tests using the solvent and the matrix. The slopes of the curves and their intercepts were compared using $t$-test.

\subsubsection{Detection and quantification limits}

LOD and LOQ were estimated using the SD of the slope and intercepts of the calibration line.

\subsubsection{Repeatability}

Repeatability was determined by repeated analysis of the conjugate sample. The migration time and CPA related to oxidize PS were evaluated. Analyses were performed using three different vials, and the content of each vial was determined four times. Repeatability was determined by comparing the values obtained according to the Horwitz equation and HorRat (Eqs. (1) and (2)) [15].

$$
\operatorname{PRSD}(\%)=2 \cdot C^{-0.1505}
$$

where PRSD is the predictive RSD and $C$ is expressed as a mass fraction.

$$
\text { HorRat }=\frac{C V_{\text {repe }}(\%)}{\frac{2}{3} P R S D_{R}}
$$

where HorRat is a theoretical value of repeatability to evaluate methods acceptance calculated using the Horwitz equation, and $\mathrm{CV}_{\text {repe }}$ is the repeatability relative standard deviation.

\subsubsection{Partial reproducibility}

Experiments were conducted on two different days by two different analysts to determine how these changes affect routine analysis. Partial reproducibility was evaluated through the intermediary standard deviation $\left(S_{i}\right)$. The results were analyzed according to the validation analytical methods guide [13], using the equation:

$S i_{(j, k)}=\sqrt{\frac{1}{2 \cdot t} \cdot \sum_{j=1}^{t}\left(y_{j 1}-y_{j 2}\right)^{2}}$

where $t$ is the total number of samples assayed, $j$ the number of samples, $k$ the number of repetitions, and $\gamma_{j 1}$ and $\gamma_{j 2}$ correspond to the first and second results obtained for the $j^{\text {th }}$ sample, respectively.

\subsubsection{Robustness}

The method's robustness was determined by electrophoretic analysis of samples under different conditions. Each change in condition was tested three times and included varying the $\mathrm{pH}$ (from 9.5 to 10.0 and 10.5) and temperature (from 35 to $40^{\circ} \mathrm{C}$ and $45^{\circ} \mathrm{C}$ ). The effect of these modifications on the retention times and CPA values for the free oxidized PS concentration was determined. The effect of variations on the method was analyzed by multiple comparisons using statistical tests (ANOVA, LSD test, $p<0.05$ ).

\subsection{Determination of PS content}

The free oxidized PS content in different batches of conjugate bulk was determinated from the slope of the calibration line of the mixture using the conjugate as the matrix. The conjugate bulks used for these analyses were MPCT 04/10, MPCT 01/11, PSC-TT 31/12, MPCT 01/12, and MPCT 02/12.

\section{Results}

\subsection{Method development}

This study was undertaken to develop a method that allows separation and quantification of free oxidized PS in a meningococcal group $\mathrm{C}$ conjugate bulk produced by BioManguinhos/Fiocruz, a Brazilian Federal Institution, and that could be used to determine the consistency of batches of conjugate bulk by evaluating the incorporation of PS during the conjugation reaction. CZE was chosen to separate free oxidized PS from the conjugate. Two peaks were observed using $20 \mathrm{mM}$ borate buffer. Free oxidized PS was completely separated from the conjugate by CZE using $50 \mathrm{mM}$ TBNa buffer, $40^{\circ} \mathrm{C}, 30 \mathrm{kV}$, and $\mathrm{pH} 10$; with migration time of $26.8 \mathrm{~min}$. Other molecules present in the conjugation reaction, such as the conjugate and activated TT, have a similar migration time (approximately $21.5 \mathrm{~min}$ (data not shown)). We next identified the peaks by spiking the conjugate sample with oxidized PS and observing its migration time (Fig. 1). Through these analyses, oxidized PS was identified as the second peak shown in the electropherogram. 


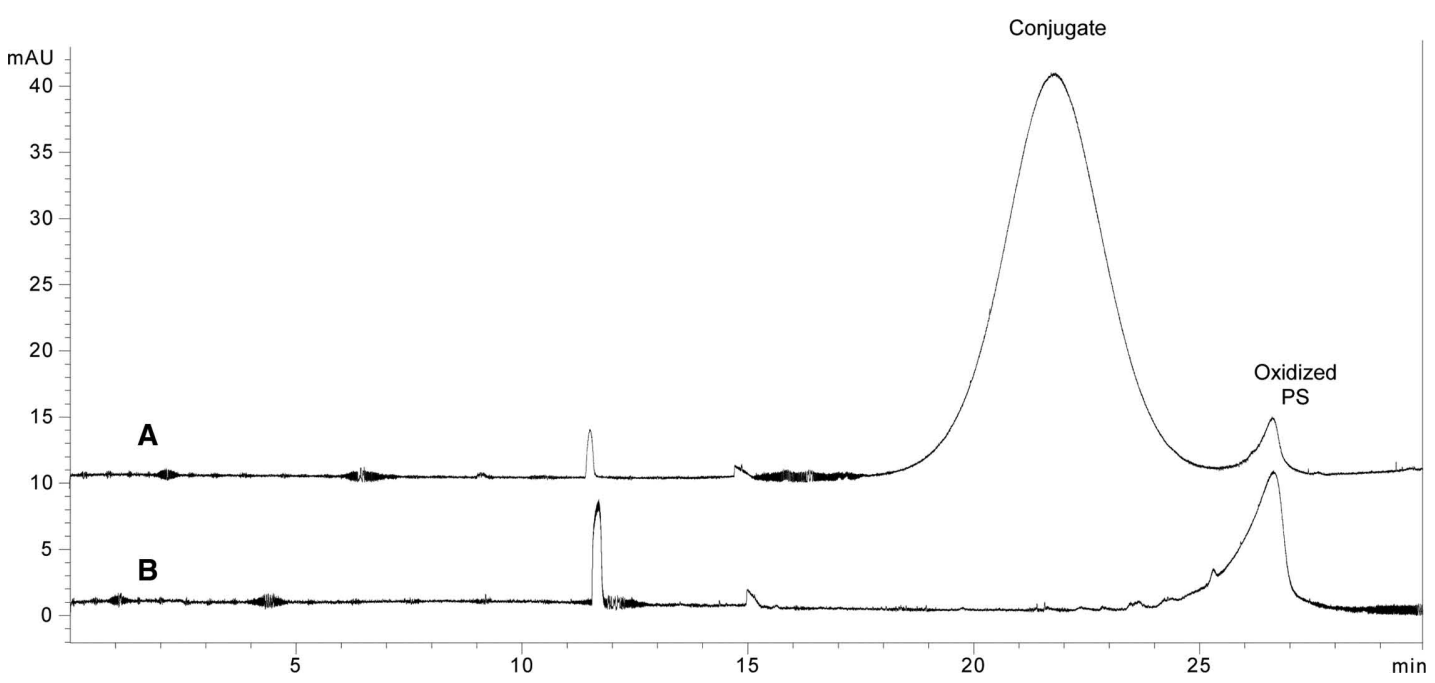

Figure 1. Overlapped electropherograms of conjugate bulk spiked with oxidized PS (A) and oxidized PS alone (B), showing the similarity in migration of the samples. Analyses were performed using a fused silica capillary, $112.5 \mathrm{~cm}(104 \mathrm{~cm}$ effective length) $\times 50 \mu \mathrm{m}$ (id), using $50 \mathrm{mM}$ TBNa, pH 10. Separation was performed using $30 \mathrm{kV}$ (normal polarity) at $40^{\circ} \mathrm{C}$. The identity of the major peaks is indicated.

A

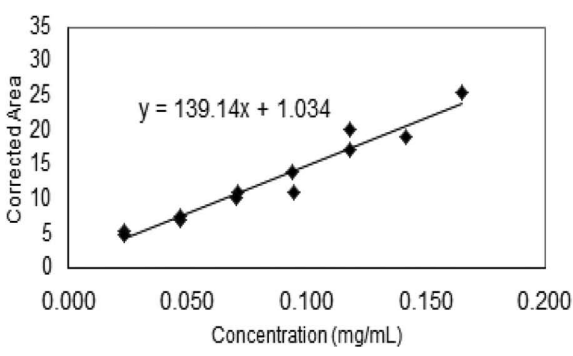

B Curve with Matrix

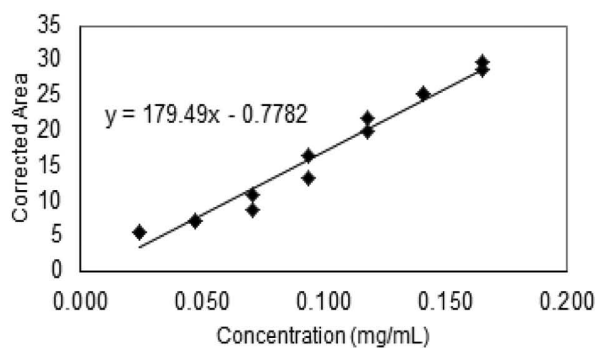

Figure 2. Oxidized PS analytical curves confectioned to evaluate the matrix effect. (A) Curve confectioned in the solvent, (B) curve confectioned in the conjugate sample.

\subsection{Calibration line preparation}

Free oxidized PS at $20 \%$ concentration was used as reference. Calibration lines were determined using seven concentrations of free oxidized PS ranging from 5 to $35 \%$ and were linear (Fig. 2).

To study the matrix effect, these same concentrations of oxidized PS were added to the conjugate. CPA values determined for oxidized PS dissolved in the matrix were subtracted from the mean CPA values of the second peak observed in the conjugate sample electropherogram. The linear (a) and angular (b) coefficients similarity between the curves confectioned in the buffer and in the sample were compared using $t$-test. The $t$-values found for linear and angular coefficients were $t_{\mathrm{a}}=2.79$ and $t_{\mathrm{b}}=3.28$, respectively. Both coefficients were higher than the acceptance criterion (2.09), indicating the influence of the matrix on the quantification. Therefore, PS was quantified by standard addition using the matrix (conjugate bulk) for calibration line confection in each batch.

We next determined the linearity of the assay. LOD and LOQ were determined as 0.0154 and $0.0454 \mathrm{mg} / \mathrm{mL}$, respectively. Because the lowest concentration $(5 \%, 0.024 \mathrm{mg} / \mathrm{mL})$ of the curve was below LOQ, only six concentrations were used to validate the method.
The linear range of the assay for quantifying free oxidized PS was between 0.047 and $0.164 \mathrm{mg} / \mathrm{mL}$, which corresponded to the interval from 10 to $35 \%$ of free PS.

\subsection{Precision}

The repeatability of the method was deduced by introducing the same amount of conjugate sample from four different vials three times and determining the migration times and CPA values of the oxidized PS peaks. The coefficients of variation were 2.02 and $2.36 \%$ for CPA and migration time, respectively. Using equations 1 and 2, the predicted relative standard deviation and the HorRat coefficient were determined for migration time as $2.015 \%$ and 0.27 , respectively, and the CPA values were 2.85 and 0.43 , respectively. These values were satisfactory as per acceptability criteria (HorRat $\leq 2)$.

Further, partial reproducibility was evaluated according to $S_{i}$, and the maximum value observed was 4.92 for $35 \%$ oxidized PS and the minimum was 1.63 for the $20 \%$ concentration. All values were satisfactory as per the acceptability criteria. 
Table 1. Quantification of free polysaccharide in some batches of conjugate bulks

\begin{tabular}{ll}
\hline Conjugate Lots & $\%$ Free PS \\
\hline MPCT 04/10 & 34.29 \\
MPCT 01/11 & 19.08 \\
PSC-TT 31/12 & $<10$ \\
MPCT 01/12 & $<10$ \\
MPCT 02/12 & $<10$ \\
\hline
\end{tabular}

\subsection{Robustness}

Robustness was evaluated by noting small variations of the electrolyte $\mathrm{pH}$ and cassette temperature during analysis, and all data showed a normal distribution according to "skewness" and "kurtosis" pattern. Variance analyses revealed that the results obtained by changing the $\mathrm{pH}$ range from 9.5 to 10.0 and 10.5 were not significantly different (with $p=0.79$ ).

For temperature evaluation, the data normality was verified using the method described above. Variance analyses of these data showed a significant difference $(p<0.05)$ between the values obtained using temperatures from 35 to $45^{\circ} \mathrm{C}$.

\subsection{Evaluation of conjugate batches}

Several batches of conjugate bulk were analyzed using the method described and validated. The values determined for free oxidized PS quantification in some of these batches are presented in Table 1 . The evaluation of these batches showed values below the limit of 20\%, except batch MPCT 04/10.

\section{Discussion}

Many disease outbreaks caused by $N$. meningitidis group C in Brazil in the last few years have been documented [16]. Bio-Manguinhos/Fiocruz has been developing a conjugate vaccine by covalent attachment of capsular PS to TT, which will be evaluated in Phase III clinical studies [1]. All production and purification steps of batches produced at different scales have been developed in the bulk production process. Thus, various methods in process control were standardized for bulk and final product to verify the presence of residues of reagents used in the conjugation step [1]. The present study developed a suitable method for the separation and quantification of free PS in the Brazilian meningococcal C conjugate vaccine using $\mathrm{CE}$.

Initially, the parameters of CE published by Lamb et al. were used [6] to further establish the optimal conditions for separating free oxidized PS from the conjugate bulk. In general, the main parameter that influenced analysis resolution was the $\mathrm{pH}$, as previously reported by Camilleri [11]. The conditions employed here allowed the detection of two peaks with a good resolution, and the second was identified as free oxi- dized PS. After separation and identification, it was possible to determine the concentration of unbound PS.

Previous studies have described mainly physicochemical and chromatographic methods for determination of free PS in conjugate vaccines $[3,17,18]$. Cuello et al. [5] studied the use of anti-TT antibodies to separate and measurefree sugar in the conjugate by molecular exclusion liquid chromatography. However, the separation results were not presented. Similarly, Cuervo et al. [19] did not present the separation profile in stability studies of a $H$. influenzae type b conjugate vaccine applying the same technique. Silveira et al. [1] also used the method described by Cuello et al. [5] to evaluate the conjugate profile used in the Brazilian meningococcal group $\mathrm{C}$ conjugate vaccine and did not observe a free PS profile. Law et al. [20] described a method to separate free PS from a conjugate vaccine against $N$. meningitidis group C using diphtheria toxoid as carrier protein. These researchers made a sample precipitation with DOC and determined the free PS content in the supernatant by colorimetric assay and ion exchange chromatography with amperometric detection.

These methods require an extraction phase and a sample preparation procedure that introduces more variables and prolongs the time of analysis $[1,20]$. Moreover, these steps hinder the methodology validation in process control.

In contrast, the $\mathrm{CE}$ methodology does not require sample preparation, uses small amounts of buffer and sample, has a high resolution power, and can be performed in a short time period. Lamb et al. [7] used CE to separate and quantify the PS of different meningococcal groups of a polysaccharide vaccine and also described the separation of free protein in some pneumococcal and meningococcal conjugate vaccines using this technique. However, these studies did not address the use of the methodology for the separation and quantification of free PS in conjugate vaccines as demonstrated in the present study.

During the methodology single validation all parameters (linearity, selectivity, repeatability, and partial reproducibility) studied were satisfactory. Moreover, a matrix effect was observed, indicating that all analysis for quantification of free PS must be performed by adding oxidized PS to the matrix (conjugate bulk). Robustness was not affected by $\mathrm{pH}$ variation; however, there were significant differences in the results when analysis was conducted at $40^{\circ} \mathrm{C}$. Therefore, the temperature should be strictly controlled, because it could reduce the reliability of the quantification of free PS.

After validating the CE method, five samples of conjugate bulk produced by Bio-Manguinhos/Fiocruz were analyzed, and the concentration of free oxidized PS in only one sample exceeded $20 \%$. Evaluation of the free sugar concentration is extremely important in the development of conjugate vaccine, because repeated administration of the $N$. meningitidis groups $\mathrm{C}, \mathrm{Y}$, and W-135 PS vaccine to adults and children has been described to induce immunological tolerance [21-24]. The results obtained in this study confirmed the suitability of $\mathrm{CE}$ for the separation and quantification of free sugar in the Brazilian meningococcal $\mathrm{C}$ conjugate vaccine. Therefore, our 
study supports the introduction of this validated method for routine quality control of vaccines batches in the future.

The work was supported by National Institute for Health Quality Control/Oswaldo Cruz Foundation and Biomanguinhos/Oswaldo Cruz Foundation.

The authors have declared no conflict of interest.

\section{References}

[1] Silveira, I. A., Bastos, R. C., Neto, M. S., Laranjeira, A. P., Assis, E. F., Fernandes, S. A., Leal, M. L., Silva, W. C., Lee, C. H., Frasch, C. E., Peralta, J. M., Jessouroun, E., Vaccine 2007, 25, 7261-7270.

[2] World Health Organization, WHO Technical Report Series, No. 932, 2004, http://whqlibdoc.who.int/ trs/WHO_TRS_932_eng.pdf.

[3] Gotschlich, E. C., Liu, T. Y., Artenstein, M. S., J. Exp. Med. 1969, 129, 1349-1365.

[4] Lei, Q. P., Shannon, A. G., Heller, R. K., Lamb, D. H., Dev. Biol. (Basel) 2000, 103, 259-264.

[5] Cuello, M., Cabrera, O., Martinez, I., Del Campo, J. M., Parajón, E., Sotolongo, F., Camaraza, M. A., Arnet, A., Suárez, M., Pérez, O., Vaccine 2006, 24, 72-73.

[6] Lamb, D. H., Summa, L., Lei, Q. P., Duval, G., Adam, O., J. Chromatogr. A 2000, 894, 311-318.

[7] Lamb, D. H., Lei, Q. P., Hakim, N., Rizzo, S., Cash, P., Anal. Biochem. 2005, 338, 263-269.

[8] World Health Organization, WHO Technical Report Series, No. 658, 1980, p29, http://whqlibdoc.who. int/trs/WHO_TRS_658_\%28part1\%29.pdf.

[9] Jessouroun, E., Silveira, I. A. F. B., Bastos, R. C., Frasch, C. E., Lee, C. H., International Patent 2005, Publication No. WO 2005/037320.
[10] Svennerholm, L., Biochim. Biophys. Acta. 1957, 241, 604.

[11] Camilleri, P., Capillary Electrophoresis. Theory and Practice, CRC Press, New York 1995.

[12] Lauer, H. H., Rozing, G. P. (Eds.), High Performance Capillary Electrophoresis, Agilent Technologies, 2009, http:// www.chem.agilent.com/Library/primers/Public/59903777EN.pdf.

[13] Instituto Nacional de Metrologia, Orientação sobre validação de métodos analíticos, 2011, DOQCGCRE-008.

[14] Agência Nacional de Vigilância Sanitária. Resolução No. 899, 2003.

[15] Horwitz, W., Pure. Appl. Chem. 1995, 67, 331-343.

[16] SIREVA II Organizacion Panamericana de la Salud, Informe Regional de SIREVA II, 2009, Washington, DC, OPS, 2010, http://www2.paho.org/hq/dmdocuments/ 2010/SIREVA\%20II\%202009.pdf.

[17] MacLennan, J., Obaro, S., Deeks, J., Williams, D., Pais, L., Carlone, G., Moxon, R., Greenwood, B., Vaccine 1999, 17, 3086-3093.

[18] Kim, J. S., Laskowich, E. R., Arumugham, R. G., Kaiser, R. E., MacMichael, G. J., Anal. Biochem. 2005, 347, 262-274.

[19] Cuervo, M. L. C., Pérez, L. R., Oviedo, M., Costa, L., Perdomo, V., Vaccine 2007, 25, 194-200.

[20] Lei, Q. P., Shannon, A. G., Heller, R. K., Lamb, D. H., Dev. Biol. (Basel, Karger) 2000, 103, 259-264.

[21] Richmond, P., Kaczmarski, E., Borrow, R., Findlow, J., Clark, S., Mccann, R., Hill, J., Barker, M., Miller, E., J. Infect. Dis. 2000, 181, 761-764.

[22] Jokhdar, H., Borrow, R., Sultan, A., Adi, M., Riley, C., Fuller, E., Baxter, D., Clin. Diagn. Lab. Immunol. 2003, $11,83-88$.

[23] Granoff, D. M., Gupta, R. K., Belshe, R. B., Anderson, E. L., J. Infect. Dis. 1998, 178, 870-874.

[24] Bröker, M., Veitch, K., Travel. Med. Infect. Dis. 2010, 8, 47-50. 